\title{
Preparation of meso-tetra(4-pyridyl)porphyrin film for optical gas sensor
}

\author{
Yu-Hui Lin ${ }^{1}$, Hui-Hsin Lin ${ }^{1}$, Chii-Wann Lin $^{1,2,3^{*}}$, Joseph Zyss ${ }^{4}$ \\ ${ }^{1}$ Institute of Biomedical Engineering, National Taiwan University, Taipei, Taiwan (10617), \\ ${ }^{2}$ Institute of Bioelectronics and Bioinformatics, National Taiwan University, Taipei, Taiwan (10617) \\ ${ }^{3}$ Institute of Applied Mechanics, National Taiwan University, Taipei, Taiwan (10617) \\ ${ }^{*}$ Corresponding author:cwlinx@ntu.edu.tw \\ ${ }^{4}$ Institut d'Alembert, Ecole Normale Supérieure de Cachan, Cachan, France
}

\begin{abstract}
The work presented a simple way to prepare a meso-tetra(4-pyridyl)porphyrin (MTPyP) film for optical gas sensor. The dip-dry coating method that was developed by our reaserch shows a strong absorption band at $418 \mathrm{~nm}$. We also proposed a simple method, peak ratio, to do the semiquantitative analysis of hydrochloric acid $(\mathrm{HCl})$ and ammonia $\left(\mathrm{NH}_{3}\right)$ molecules adsorbed on the dipdry coating MTPyP film by their absorption bands. The MTPyP film was prepared on glass substrate and spectrometer was used to obtain the spectra as sensing the vapor molecules. The ratio of peaks values at $418 \mathrm{~nm}$ and $450 \mathrm{~nm}$ was used to identify the vapor types and to determine the concentrations. The results show that the peak ratio of $\mathrm{HCl}$ is approaching less than one and the peak ratio of $\mathrm{NH}_{3}$ is always larger than one for two-hour measurment.
\end{abstract}

Key words: MTPyP film optical gas sensor, hydrogen chloride/ammonia detection, dip-dry coating, peak ratio

\section{Introduction}

In recent years, porphyrin complexes have become one of hot research topics for developing gas sensors and optical sensors because of its advantages of light sensitivity, chemical and thermal stability, and easy modifycation. The optical properties of porphyrins would change as specific gases combined with porphyrin molecules, like $\mathrm{NO}_{2}, \mathrm{NO}, \mathrm{CO}, \mathrm{HCl}$, which are based on a rich $\pi$-electron system. Hence, some optical methods, such as the ultraviolet, infrared, fluorescence, phosphorrescence and Raman spectroscopy, could be used to detect the changes of optical properties of porphyrins [1]. Therefore, porphyrin complexes becomes an ideal sensing model to study the porphyrin-based sensors. Various techniques have been proposed to prepare the porphyrin films, such as self-assembled monolayer [2], Langmuir-Blodgett Technique [3], and vacuum deposition [4], but these techniques are so complicated for practical application since it takes a lot of time. At same time, these techniques require expensive equipment, which lead to higher costs. Additionally, the optical gas sensors also suffer the problem of drifting baselines, which is also the problems for most optical sensors.

To solve the above problems, a simple method for preparing the Meso-tetra(4-pyridyl) porphyrin(MTPyP) thin film which called dip-dry method was proposed. Then the UV-VIS spectrometer was used to obtain their characteristics of absorption spectra of the MTPyP dip-coating film as sensing $\mathrm{NH}_{3}$ and $\mathrm{HCl}$ molecules. In addition, we also proposed a simple method, peak ratio, to do the semiquantitative analysis of $\mathrm{HCl}$ and $\mathrm{NH}_{3}$ molecules adsorbed on the dip-dry coating porphyrin film.

\section{Materials and Methods}

\subsection{Fabrication of MTPyP thin film}

$1.25 \mathrm{mM}$ and $1 \mathrm{mM}$ MTPyP (Frontier Scientific) solutions were prepared individually in $1 \%$ and $10 \%$ toluene (TEDIA) solutions that were a mixture of ethanol (Anhydrous) and toluene with a volume ratio of 99 to 1 and of 9 to 1 , respectively. In spin-coating, a $50 \mu$ drop of $1.25 \mathrm{mM}$ MTPyP was spread out by the spincoater (Laurell) to form a thin film on glass surface at a speed of $800 \mathrm{rpm}$ for the first $10 \mathrm{~s}$ and then $2000 \mathrm{rpm}$ for $40 \mathrm{~s}$. In the dip coating methods, $1.25 \mathrm{mM}$ MTPyP with a volume of $12 \mathrm{ml}$ was deposited directly on glass surface to form a thick film. Finally, in the method of dipdry coating, a $50 \mu \mathrm{l}$ drop of $1 \mathrm{mM}$ MTPyP was dropped on glass surface, and then the specimen was treated by nitrogen gas to remove the residual MTPyP solution.

\subsection{Instrumentation}

The absorption spectra of MTPyP film and gaseous detection were carried out by a UVVIS spectrometer (Varian) at the room temperature. Two handmade acrylics cell with 
volume of 22.5 and $30 \mathrm{~cm}^{3}$ are for static gas sensing. Glass slide is the substrate.

\subsection{Sensing procedure of MTPyP thin film}

At first, the $25 \% \mathrm{NH}_{3}$ and $37 \% \mathrm{HCl}$ liquid without dilution was individually put into the handmade acrylics cell with a volume of $30 \mathrm{~cm}^{3}$ and then sealed the cell in the measurment process. The optical absorption of the MTPyP thin film ranged from 370 to $500 \mathrm{~nm}$ was continuously measured until the vapour pressure of the cell reached to dynamic equilibrium state at room temperature.

The absorption spectra of MTPyP thin films were also investigated by static measurment after adding $5 \mathrm{ul}$ drops of $\mathrm{HCl}$, and $\mathrm{NH}_{3}$ with the lower concentration of $3 \mathrm{M}$ to a cell with volume of $22.5 \mathrm{~cm}^{3}$ individually. The detection time was over 20 minutes.

\section{Result and discussion}

\subsection{The characterization of MTPyP films produced by different methods}

The absorption spectra of MTPyP films prepared by spin-coating, dip-coating and dip-dry coating are shown in Fig. 1 and the absorption bands, Soret bands, are 425, 419, $418 \mathrm{~nm}$, respectively. The film prepared by spin-coating presents a weaker intensity than the other two methods. The spin-coated film is not uniform, because solvent with high evaporation rate contributed to the non-uniform films. The highly volatile characteristic of MTPyP in ethanoltoluene mixture, which prone to completely vaporized during spin-coating process, may dry up too fast to form a film. The dip-dry coated film presents a stronger intensity than the other two methods, because less volume and rarer concentration of MTPyP solution for dip-dry coating could obtain a uniform film after removing the residual solution by $\mathrm{N}_{2}$ gas.

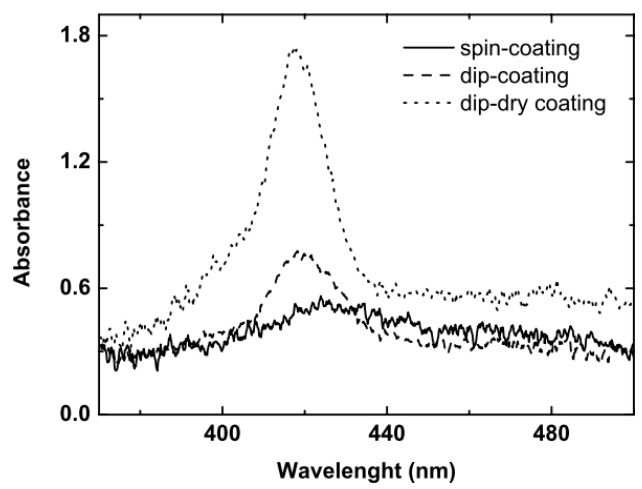

Fig. 1. Absorption spectra of MTPyP thin films prepared by spin-coating, dip-coating and dip-dry coating, and concentrations of MTPyP are 1.25, 1.25 and $1 \mathrm{mM}$, respectively.
3.2 Sensing of $\mathrm{NH}_{3}$ and $\mathrm{HCl}$ gas using MTPyP dip-dry coating film
In the experiment of $\mathrm{NH}_{3}$ detection, Fig. 2(a)

shows the absorption spectra of the MTPyP film exposed to $\mathrm{NH}_{3}$ vapor. The solid black line with an absorption peak of $418 \mathrm{~nm}$ is the original spectrum without exposing to $\mathrm{NH}_{3}$ vapor. Then, $10 \mathrm{ml}$ of ammonia solution with a concentration of $25 \%$ was added into a transparent cell with a volume of $30 \mathrm{~cm}^{3}$ where a MTPyP film had been placed in advance. The intensity of absorption spectra at $418 \mathrm{~nm}$ decreased during $0 \mathrm{~s}$ to $1418 \mathrm{~s}$. Decreasing intensity of absorption peak at $418 \mathrm{~nm}$ induced by $\mathrm{NH}_{3}$ gaseous molecules might cause by the electrostatic force between the ammonia molecules and prophyrin, since the porphyrin we adopted here without cental metal ion. Moreover, only the intensity of Soret band decreased as more ammonia was evaporating to the air and no Soret red-shifting occured in this result, which indicats that no electron transfer occured between ammonia and porphyrin molecules.

(a)

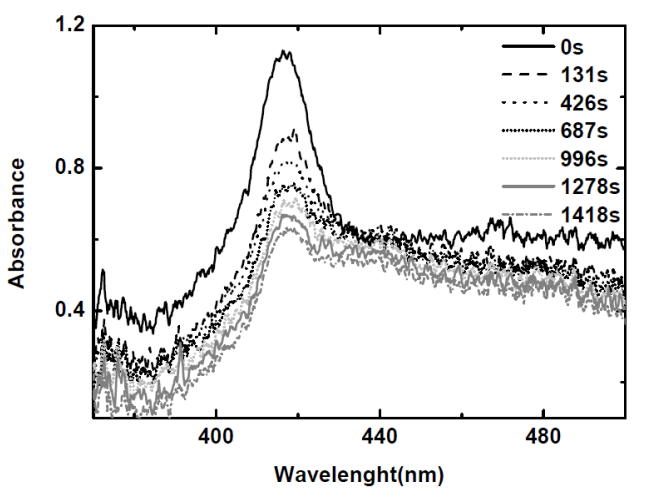

(b)

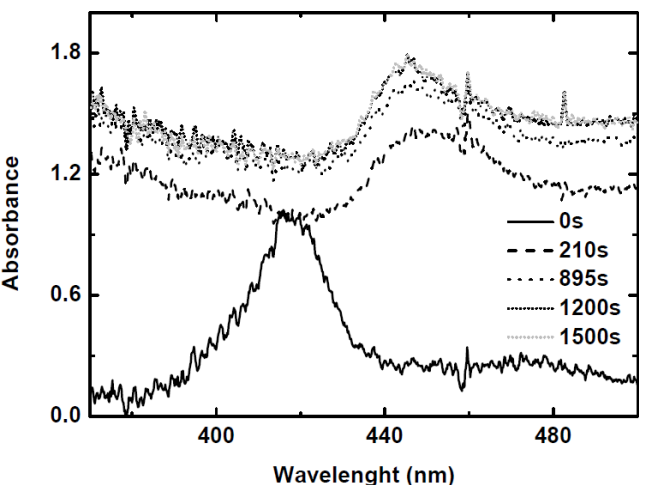

Fig.2. (a) Absorption spectral changes of MTPyP film by exposing to $\mathrm{NH}_{3}$ gas (b) Absorption spectral changes of MTPyP film by exposing to $\mathrm{HCl}$ gas

In Fig. 2(b), the solid black line is the spectrum of MTPyP film before exposing to any $\mathrm{HCl}$ gas. The absorption bands, Soret band, is 418 $\mathrm{nm}$. Then, $1.7 \mu \mathrm{l}$ drop of hydrochloric acid with a concentration of $37 \%$ without dilution was put into a small transparent cell with a volume of 30 $\mathrm{cm}^{3}$ where a MTPyP film had been placed in advance, and the spectra ranged from of 350 $\mathrm{nm}$ to $500 \mathrm{~nm}$ were obtained with time series. During this period, the hydrochloric acid molecules evaporated and attached onto this solid state sensor of MTPyP film. 
After approximately $200 \mathrm{~s}$, the results of this experiment were observed. The intensity of Soret band decreased due to more and more hydrochloric acid molecule absorbed, and the Soret band shifted from $418 \mathrm{~nm}$ to $450 \mathrm{~nm}$. However, at the time of 15 minutes, 900 seconds, the absorption band had a blue shift to $445 \mathrm{~nm}$, which might be caused by the interference of water vapor that continued evaporating before reaching the state of vapor pressure. Finally, the position of the Soret band at $445 \mathrm{~nm}$ were not shifted after approximately 20 minutes, when hydrochloric acid solution and vapor reach to dynamic equilibrium of vapor-liquid phase. Therefore, the response time for the Soret band shifting toward $445 \mathrm{~nm}$ highly depends on the concentration of hydrochloric acid solution. Moreover, the red shift of Soret band was caused by the attachment of proton on the peripheral pyridyl groups of porphyrin and the inner nitrogen atoms of pyrrole ring [6]. The relative intensity of Soret band at $418 \mathrm{~nm}$ become smaller than the Soret band before exposing to hydrochloric acid molecules, and the absorption of spectra also became higher as more proton evaporated into air.

3.3 The semi-quantitative analysis of $\mathrm{NH}_{3}$ and $\mathrm{HCl}$ gas using MTPyP films

In Fig. 2 (b), the absorption unit of $418 \mathrm{~nm}$ at $210 \mathrm{~s}$ is overlap with the original spectrum, but its Soret band had shifted to $450 \mathrm{~nm}$. Hence, the quantitative analysis is not enough to determine the variance of absorption intensity and the shifting of Soret band. Furthermore, the determination of the precise response time of MTPyP film to $\mathrm{HCl}$ molecule should carry out by removing the interference of water vapor in the future. Although the experiment demonstrated here is simple, it could reflect the practical circumstance as the acid vapor leaks in a closed space. Although the intensity and the position of Soret band of porphyrin would change as $\mathrm{HCl}$ and $\mathrm{NH}_{3}$ absorbed, the baseline of every spectrum did not vary regularly with increasing the concentration of vapor molecules. In Fig. 2, we could observe that the first two spectra exposed to the $\mathrm{HCl}$ molecules were higher than the original spectrum (the solid black line), but the other spectra were lower than the black line. However, the quantitative method to locate the variance of Soret band and then to compare with the original spectrum is not enough for the purpose of detecting proton in real time. For the detection of $\mathrm{NH}_{3}$ molecules, the variance of spectra was not complicated, but the baselines were also drifting as more molecules attached. Therefore, we proposed to utilize the ratios of peaks values at $418 \mathrm{~nm}$ and $450 \mathrm{~nm}$ for every spectrum to identify the types and to roughly determine the concentrations of vapor molecule. The peak ratio $\left(A_{418} / A_{450}\right)$ is defined as the ratio of the absorbance bands at $418 \mathrm{~nm}$ and $450 \mathrm{~nm}$ in the same spectrum.

(a)

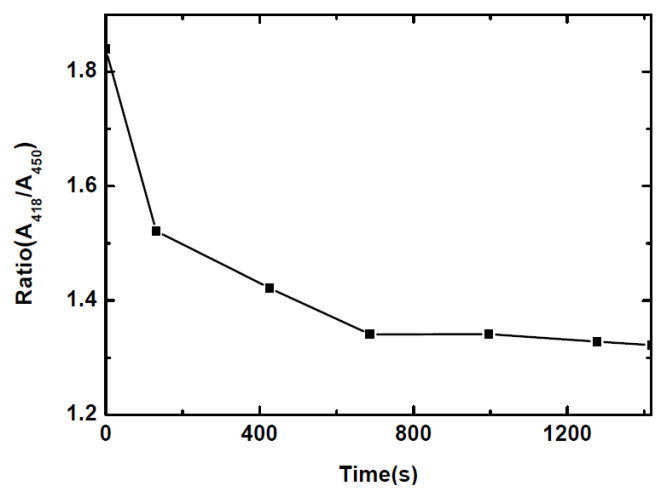

(b)

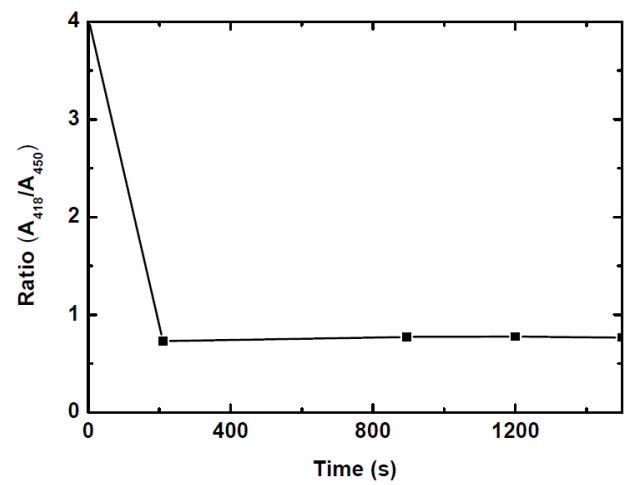

Fig.3. (a) Peak ratio of $418 \mathrm{~nm}$ and $450 \mathrm{~nm}$ changed with time obtained from Fig.2.(a); (b) Peak ratio of $418 \mathrm{~nm}$ and $450 \mathrm{~nm}$ changed with time obtained from Fig.2.(b).

The value of peak ratio was small than 1 , after exposing to $\mathrm{HCl}$ gas (Fig. 3(a)). Due to the red shift of Soret band to $450 \mathrm{~nm}$, the value approached steady as more molecules attached on the MTPyP film. The value of peak ratio closed to 1, after exposing to $\mathrm{NH}_{3}$ gas (Fig. $3(b))$. After two hours, the value of peak ratio approached 1.3. Hence, the differences values of peak ratio of $418 \mathrm{~nm}$ and $450 \mathrm{~nm}$ in the spectra of sensing $\mathrm{HCl}$ and $\mathrm{NH}_{3}$ were obvious. We could obtain the value of peak ratio for $\mathrm{HCl}$ was less than 1 , and the value of peak ratio for $\mathrm{NH}_{3}$ was always larger than 1 . This result indicated that the potential of porphyrin-based optical gas sensor provided a simple method to identify $\mathrm{HCl}$ and $\mathrm{NH}_{3}$ gas.

\subsection{Sensing of $\mathrm{NH}_{3}$ and $\mathrm{HCl}$ gas at lower} concentration

In fig.4 (a) and (b), we could found that the lower concentration of $\mathrm{NH}_{3}$ and $\mathrm{HCl}$ solutions were difficult be evaporated into air, so the variance of the spectra changed with time were not as apparent as those in high concentration. According to the results, the related intensity of absorption spectra for $\mathrm{NH}_{3}$ almost didn't change. However, only the $\mathrm{H}^{+}$from the $\mathrm{HCl}$ gas caused 
an obvious red shift, from $418 \mathrm{~nm}$ to $450 \mathrm{~nm}$. The MTPyP film showed the better sensitivity to $\mathrm{HCl}$ gas compared with that of $\mathrm{NH}_{3}$ gas.

In fig.5 (a), the value of peak ratio was approaching to 2 , due to the relative intensity of the Soret band at $418 \mathrm{~nm}$ was almost constant (fig.4 (a)). The sepctra after $90 \mathrm{~s}$ were almost unchanged during by exposing to less $\mathrm{NH}_{3}$ gas. However, in fig.5 (b), the value of peak ratio was also smaller than 1 , as the Soret band exposed to $\mathrm{HCl}$ gas. This results showed that the slope of the peak ratio before 400 seconds did not change dramatically.

These results indicated that the use of MTPyP dip-dry coating films for the development of optical gas sensor had the advantages for detection of $\mathrm{HCl}$, whether the porphyrin thin films in high concentration or low concentration, moreover, they showed the poor sensitivity in lower ammonia concentration.

(a)

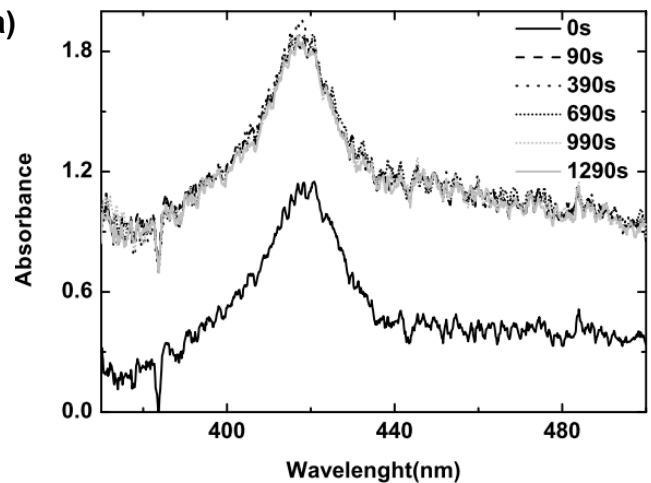

(b)

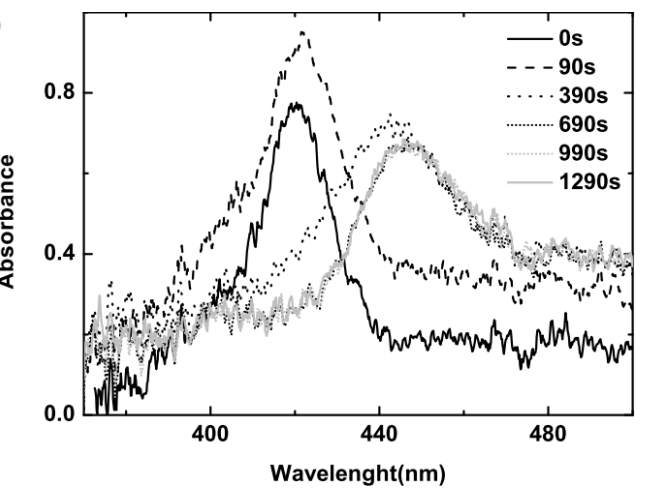

Fig.4. The spectra for sensing various acid/basic vapor, (a) $\mathrm{NH} 3$;(b) $\mathrm{HCl}$. The concentrations of solutions were all $3 \mathrm{M}$.

(a)

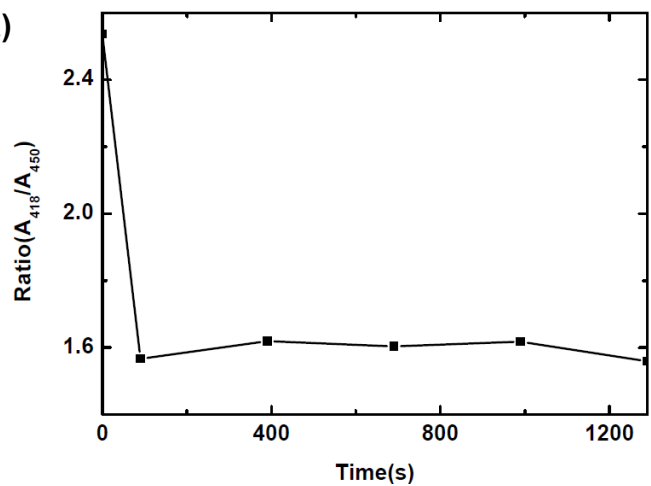

(b)

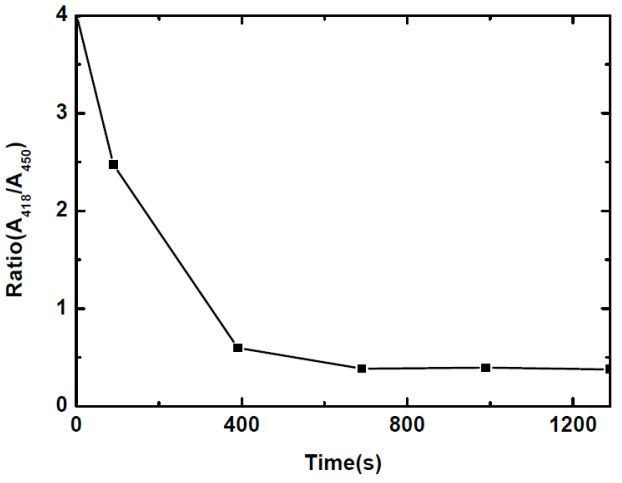

Fig.5. (a) Peak ratio of $418 \mathrm{~nm}$ and $450 \mathrm{~nm}$ changed with time obtained from Fig.4.(a); (b) Peak ratio of $418 \mathrm{~nm}$ and $450 \mathrm{~nm}$ changed with time obtained from Fig.4.(b).

\section{Conclusions}

This work demonstrated a simple way to prepare MTPyP films for $\mathrm{NH}_{3}$ and $\mathrm{HCl}$ gas sensor, and we also presented a semiquantitative analysis of $\mathrm{NH}_{3}$ and $\mathrm{HCl}$ gas using MTPyP films. The results demonstrated that this MTPyP film shows a good potential to identify the $\mathrm{HCl}$ and $\mathrm{NH}_{3}$ gas at room temperature.

\section{Acknowledgements}

This work was supported by the National Science Council (NSC) of Taiwan under contract No. NSC-NSC 99-2221-E-002 -117 MY3.

\section{References}

[1] D.B. Papkovsky, Luminescent porphyrins as probes for optical (bio)sensors, Sens. Actuators B, vol. 11, pp. 293-300 (1993); doi: 10.1016/0925-4005(93)85267-E

[2] Z.Zang, S.Hou, Z.Zhu, Z.Liu, Preparation and characterization of a porphyrinself-assembled monolayer with a controlled orientation on gold, Langmuir 16, 537-540 (2000); doi: 10.1021/la990570g

[3] A.Ulman, An introduction to ultrathin organic films from langmuir-blodgett films to selfassembly, AcademicPress, San Diego, CA (1991); doi: 10.1002/ange.19921040445

[4] K.Nakamura, M.Watanabe, M.Zhou, M.Fujishima, M.Tsuchiya, T.Handa, S.Ishii, H.Noguchi, K.Kashiwagi, and Y.Yoshida, Plasma polymerization of cobalt tetraphenylporphyrin and the functionalities of the thin films produced, Thin Solid Films, vol.345, issue 1, pp. 99-103 (1999); doi: 10.1016/S0040-6090(99)00093-0

[5] P.Muthukumar, S.Abraham John, Highly sensitive detection of $\mathrm{HCl}$ gas using a thin film of meso-tetra(4-pyridyl)porphyrin coated glass slide by optochemical method, Sens. Actuators B, vol. 159, pp. 238-244 (2011); doi: 10.1016/j.snb.2011.06.079 